\title{
Há envolvimento dos músculos cervicais na patogênese da migrânea e da cefaleia do tipo tensional? Uma revisão
}

\author{
Are cervical muscles involved in migraine and tension-type headache \\ pathogenesis? A review
}

\author{
Débora Wanderley Bezerra e Silva', Thaís Ferreira Lopes Dinis Maia', \\ Camila Caroline Silva de Almeida', Carlanne do Rêgo Barros Lacerda', Juliana Elias de Albuquerque ${ }^{1}$, \\ Joaquim José de Souza Costa Neto², Gisela Rocha Siqueira', \\ Alberto Galvão de Moura Filho', Luciana Rodrigues Belo², Daniella Araújo de Oliveira ${ }^{1,2}$ \\ 'Departamento de Fisioterapia, Universidade Federal de Pernambuco - Recife, PE, Brasil \\ 2Programa de Pós-Graduação em Neuropsiquiatria e Ciências do Comportamento, \\ Universidade Federal de Pernambuco - Recife, PE, Brasil
}

Bezerra e Silva DW, Maia TF, Almeida CC, Lacerda CR, Albuquerque JE, Costa Neto JJ, Siqueira GR, Moura Filho AG, Belo LR, Oliveira DA. Há envolvimento dos músculos cervicais na patogênese da migrânea e da cefaleia do tipo tensional? Uma revisão. Headache Medicine. 2013;4(2):70-6

\section{RESUMO}

A fisiopatologia da migrânea e da cefaleia do tipo tensional (CTT) ainda é pouco conhecida e controversa. Estudos sugerem uma associação entre a presença destes tipos de cefaleia e os desequilíbrios na musculatura flexora profunda e superficial da cervical. Desta forma, poder-se-ia suspeitar que a presença destas alterações estruturais e comportamentais da musculatura cervical estaria associada de alguma forma à patogênese da migrânea e da CTT, promovendo modificações na biomecânica da cabeça e região cervical, bem como limitações na mobilidade cervical. objetivo deste estudo foi revisar as publicações mais relevantes sobre o papel da musculatura cervical na patogênese da migrânea e da CTT, a fim de fundamentar e direcionar o tratamento não farmacológico de pacientes com desordens musculares associadas a estas doenças. Foi feito um levantamento da literatura, entre janeiro/2012 e junho/ 2013, nas bases de dados PubMed, Cochrane e Bireme. Os descritores utilizados foram: 'transtornos de enxaqueca', 'cefaleia do tipo tensional', 'músculos do pescoço', 'ultrassonografia', 'eletromiografia', e seus equivalentes em inglês. Foram identificados 73 artigos, dos quais 8 foram excluídos, segundo os critérios de elegibilidade. Evidências sugerem o envolvimento de mecanismos periféricos provenientes dos músculos cervicais na sensibilização do sistema nervoso central e no surgimento da migrânea e da CTT, indicando que ambas podem apresentar alterações musculoesqueléticas cervicais. Entretanto, a maioria destes estudos é baseada em modelos experimentais com animais, os quais apresentam sistemas de processamento da dor diferentes dos humanos. Além disso, os aspectos metodológicos destas pesquisas comprometem a evidência encontrada nos resultados.

Palavras-chave: Transtornos de enxaqueca; Cefaleia do tipo tensional; Músculos do pescoço; Ultrassonografia; Eletromiografia

\section{ABSTRACT}

Migraine and tension-type headache (TTH) physiopathology is still unclear and controversial. According to researches, there is an association between these primary headaches and musculoskeletal dysfunctions of deep and superficial cervical flexor muscles. Therefore, it is possible to suspect that these structural and behavioral muscles dysfunction are associated with migraine and CTT pathogenesis, causing some changes in head and neck biomechanics, as well as limitations in cervical mobility. Thus, the need for more information required a review of more relevant studies to clarify the role of neck muscles in migraine and CTT pathogenesis, in order to support and direct the nonpharmacological treatment of patients with headache and muscular disorders. The Pubmed, Cochrane and Bireme databases were searched, between January/2012 and June/ 2013, using the keywords: 'migraine disorders', 'tension-type 
headache', 'neck muscles', 'ultrasound', 'electromyography'. The selection identified 73 articles, of which 8 were excluded according to the eligibility criteria. Evidences suggest the existence of a cause and effect relationship between cervical structures and migrainous and TTH pain, indicating that both peripheral and central mechanisms of sensitization are involved. However, most of these studies are based on experimental animal models, which have different painprocessing systems from humans. Furthermore, the methodological aspects decrease the strength of evidence found in their results.

Keywords: Migraine disorders; Tension-Type headache; Neck muscles; Ultrasonography; Electromyography

\section{INTRODUÇÃO}

Segundo a Organização Mundial de Saúde (OMS), a cefaleia é uma das principais razões para o indivíduo procurar um médico.(1) Dentre os diversos tipos existentes, as cefaleias primárias são consideradas pela OMS como um problema importante de saúde pública devido à elevada prevalência na população mundial e ao impacto relevante na sociedade. ${ }^{(2)}$

Com base em estimativas da prevalência global de pessoas com transtornos da cefaleia, achados da literatura indicam que a dor de cabeça representa a terceira queixa mais comum nos ambulatórios médicos. ${ }^{(3)}$ Além disso, estima-se que $46 \%$ da população mundial apresentaram um episódio do sintoma no último ano e $64 \%$ já tiveram alguma crise de cefaleia na vida. ${ }^{(2)}$

A migrânea e a cefaleia do tipo tensional (CTT) são as cefaleias primárias mais frequentes, caracterizadas clinicamente por crises recorrentes e manifestações associadas. ${ }^{(4)}$ Com mecanismos controversos e fisiopatologias complexas e ainda pouco esclarecidas, estes tipos de cefaleia são agravados por fatores desencadeantes, envolvendo processos centrais, de alteração antinociceptiva, e periféricos, de acometimento muscular. Contudo, não se sabe até que ponto tais fatores contribuem para o desenvolvimento destes tipos de dor de cabeça. ${ }^{(5)}$

Neste contexto, é amplamente divulgado em estudos que as disfunções musculoesqueléticas cervicais podem ter um papel importante na origem da cefaleia. ${ }^{(6)} \mathrm{A}$ convergência de vias cervicais superiores e vias trigeminais nociceptivas permite que a dor do pescoço seja referida nas áreas inervadas pelo nervo trigêmeo na face e na cabeça. Desta forma, a dor no segmento cefálico poderia ser uma dor referida da coluna cervical ou dos tecidos moles do pescoço(7) e a capacidade funcional alterada e prejudicada da musculatura cervical poderia ser tanto a causa quanto a consequência das cefaleias. ${ }^{(6)}$

Todavia, existem divergências na literatura se a dor causa mudanças no controle motor da coluna vertebral ou se as alterações musculoesqueléticas da coluna desencadeiam a dor. ${ }^{(8)}$ Ademais, apesar de alguns estudos apontarem a presença de alterações musculoesqueléticas cervicais em pacientes com migrânea e CTT, devido à baixa qualidade metodológica dos mesmos, há evidências insuficientes sobre o tema.

Observa-se ainda que as investigações acerca deste assunto são realizadas em populações não homogêneas, muitas vezes em idades mais avançadas, nas quais é mais frequente a presença de doenças crônicas, cujos impactos podem contribuir para o surgimento das disfunções musculoesqueléticas, tornando questionável a associação de determinados aspectos à cefaleia. Tais achados apontam para a importância do conhecimento dos fatores associados à cefaleia e do desenvolvimento de estudos com maior rigor metodológico em seres humanos.

Em decorrência da lacuna existente, o presente estudo iniciou-se a partir de suspeitas levantadas na literatura sobre os possíveis fatores associados ao surgimento da migrânea e da CTT. Assim, o objetivo deste estudo foi revisar os estudos mais relevantes relativos à elucidação do papel da musculatura cervical na patogênese da migrânea e da CTT, a fim de fundamentar e direcionar o tratamento fisioterapêutico de pacientes com desordens musculares associadas a estas afecções.

\section{MÉTODOS}

Foi feito um levantamento da literatura, no período de janeiro de 2012 a junho de 2013, nas bases de dados PubMed, Cochrane e Bireme, utilizando os descritores 'transtornos de enxaqueca', 'cefaleia do tipo tensional', 'músculos do pescoço', 'ultrassonografia', 'eletromiografia', além de seus equivalentes em inglês. Os limitadores foram: artigos nos idiomas inglês, francês, espanhol e português.

A análise utilizada nesta revisão avaliou os seguintes aspectos nos estudos: 1 - análise do papel da musculatura cervical na patogênese da migrânea e da cefaleia tipo tensional; 2 - análise das alterações musculoesqueléticas na migrânea e na cefaleia tipo tensional; 3 - análise dos métodos de avaliação das disfunções musculoesqueléticas cervicais em pacientes com cefaleia. 


\section{REVISÃO DE LITERATURA}

\section{O papel da musculatura cervical na patogênese da migrânea e da cefaleia tipo tensional}

A fisiopatologia da migrânea e da CTT ainda é pouco conhecida e controversa. ${ }^{(9)}$ Pesquisas mostram que a origem destas cefaleias é multifatorial ${ }^{(10-12)}$ e ocorre tanto a partir de mecanismos periféricos, como as alterações nos tecidos miofasciais, quanto de mecanismos centrais encefálicos. ${ }^{(10,13-15)}$

Durante muitos anos acreditava-se que o mecanismo envolvido na CTT era decorrente da contração aumentada e prolongada dos músculos da cabeça, pescoço, ombros e face. Por consequência, a contração induzia uma isquemia muscular, na qual eram produzidas e liberadas substâncias algogênicas. No entanto, estudos mais atuais evidenciaram que a fisiopatologia da CTT envolve um aumento dos estímulos aferentes nociceptivos sobre um sistema no qual a modulação da dor já é alterada, sugerindo a existência de mecanismos centrais e periféricos na patogênese da CTT. ${ }^{(16)}$

Por outro lado, a existência de fatores que promovam uma contração acentuada dos músculos pericranianos contribui para o agravamento ou precipitação da cefaleia. Desta forma, o surgimento da dor é associado a um incremento nos inputs nociceptivos sobre os tecidos miofasciais pericranianos, em decorrência da sensibilização de vias aferentes sensitivas periféricas. ${ }^{(10,12,16-20)}$

Observa-se, portanto, que os mecanismos de sensibilização periférica podem desencadear a sensibilização central, através do prolongamento da estimulação nociceptiva. $\bigcirc$ acúmulo de estímulos nociceptivos promove a sensibilização dos neurônios supraespinhais de segunda e terceira ordens, bem como a diminuição da inibição ou facilitação aumentada da transmissão nociceptiva no corno dorsal da medula. ${ }^{(10,12,20)}$ Por consequência, este processo de sensibilização resulta na conversão da CTTE em CTTC..10, 21)

Além disso, o acúmulo de impulsos nociceptivos nos tecidos periféricos pode induzir alterações no comportamento dos neurônios do corno dorsal,(22) favorecendo o surgimento de pontos-gatilho (PG) miofasciais. (23) Por sua vez, a presença dos PG ativos é responsável pelas queixas de dor, devido ao aumento dos níveis de substâncias algogênicas liberadas nos tecidos. ${ }^{(24)}$
Já a migrânea é percebida dentro dos dermátomos do nervo trigêmeo e dos nervos cervicais superiores (C2, C3), o que requer a ativação do núcleo trigeminocervical. ${ }^{(15)}$ Este núcleo localiza-se na porção superior da medula espinhal, no qual convergem as fibras sensitivas do nervo trigêmeo e as fibras sensitivas das raízes nervosas cervicais superiores. ${ }^{(25)}$ Portanto, sugere-se que as estruturas cervicais nele situadas podem estar envolvidas na patogênese da migrânea. ${ }^{(15)}$

Evidências atuais mostram que descargas aferentes das estruturas cervicais com disfunção podem desencadear a dor craniocervical associada à migrânea, através da sensibilização central do núcleo trigêminocervical. ${ }^{(26-30)}$ Os estímulos mantidos e prolongados, enviados pelas fibras aferentes nociceptivas A-delta e C, ${ }^{(31-33)}$ podem evocar mudanças em neurônios de segunda e terceira ordens, localizados no núcleo trigêmino-cervical|(34) e no tálamo, respectivamente. ${ }^{(35)}$ Posteriormente os estímulos são transmitidos a regiões corticais de percepção da dor, ${ }^{(35)}$ facilitando os ataques migranosos. ${ }^{(36,37)}$

As fibras nociceptivas são dotadas de receptores para diversas substâncias algogênicas, ${ }^{(33)}$ as quais são liberadas durante a migrânea pelo núcleo caudal do trigêmeo, produzindo vasodilatação, aumento da permeabilidade vascular e o consequente extravasamento de proteínas e ativação plaquetária. ${ }^{(30)}$ Este processo resulta em dor pulsátil e sensibilização periférica, características deste tipo de cefaleia. ${ }^{(30)}$

No entanto, os modelos teóricos que sugerem que os inputs aferentes cervicais são capazes de facilitar a dor na migrânea são baseados em estudos com animais. ${ }^{27,38)}$ Assim, devido às diferenças anatômicas entre os sistemas de processamento da dor entre as espécies, é necessário que os dados encontrados nestes estudos sejam interpretados com cautela. ${ }^{(15)}$

\section{Alterações musculoesqueléticas na migrânea e cefaleia tipo tensional}

Em decorrência dos mecanismos centrais e periféricos, anormalidades musculoesqueléticas cervicais têm sido frequentemente relacionadas tanto aos pacientes migranosos, quanto aos com CTT. ${ }^{(39-42)}$ Assim, pesquisas são realizadas com o intuito de evidenciar quais são os principais músculos envolvidos e as alterações mais encontradas em pacientes com cefaleia para fundamentar e direcionar o tratamento das desordens musculares associadas a estas doenças. ${ }^{(43)}$ 
Neste sentido, estudos revelaram que diferentes músculos cervicais contribuem para a estabilização cervical e podem estar comprometidos em pacientes com dor, como é o caso da dor cervical crônica e da cefaleia. No entanto, apesar do grande número de músculos avaliados, a musculatura flexora profunda cervical destaca-se por desempenhar uma função postural, auxiliando na manutenção do alinhamento cervical. ${ }^{(44-46)}$

Vários autores concordam que em pacientes com dor cervical crônica observa-se a presença de disfunções na musculatura flexora profunda da cervical, como os músculos flexor longo da cabeça (FLC) e o flexor longo do pescoço (FLP).. ${ }^{(44,47-49)}$ A partir dos achados, poderse-ia suspeitar que as disfunções encontradas nesta musculatura também podem estar presentes em pacientes com cefaleia. ${ }^{(47)}$ Todavia, há uma escassez de pesquisas avaliando a musculatura flexora profunda da cervical em pessoas com migrânea e CTT.

Em relação a outras disfunções encontradas em pacientes com cefaleia, estudos mostram que pacientes com CTTE e CTTC apresentam um aumento da tensão pericranial. ${ }^{(39)}$ Já os pacientes com migrânea, além do aumento da tensão dos músculos pericraniais, apresentam um aumento da sensibilidade à palpação muscular e maior número de trigger points. ${ }^{(41)}$

Além disso, a postura de anteriorização da cabeça é frequentemente observada em pacientes com migrânea, CTTE ou CTTC. $(9,24,41,50)$ Por outro lado, alguns autores consideram que a mobilidade cervical pode estar limitada nestes pacientes. ${ }^{(40)}$

\section{Avaliação das disfunções musculoesqueléticas cervicais em pacientes com cefaleia}

Devido à importância da musculatura flexora profunda da cervical, há na literatura uma grande variedade de estudos que avaliam esta estrutura por meio de diferentes técnicas. Porém, por serem músculos muito profundos, os métodos tradicionais, como a palpação e o teste de força muscular, não são eficazes. ${ }^{(44)}$

Outros meios permitem avaliar músculos profundos da cervical, dentre eles a ultrassonografia (USG) e a ressonância magnética (RM). ${ }^{(51-52)}$ Considerada como padrão-ouro, a RM é o método mais eficaz para determinar o tamanho do músculo, ${ }^{(53)}$ no entanto, devido aos seus altos custos, não é acessível a toda população. ${ }^{(44)}$

Por outro lado, a USG é um procedimento de baixo custo, que tem a vantagem de não ser invasivo, ${ }^{(44,53)}$ podendo ser utilizado para garantir a segurança e o conforto dos participantes de pesquisas. Desta forma, a USG apresenta-se como uma alternativa de avaliação, útil para direcionar o tratamento de pacientes com disfunções na musculatura flexora profunda da cervical. ${ }^{(53)}$

No exame de USG é possível avaliar os músculos da coluna durante repouso e atividade, além de permitir a obtenção de medidas diretas dos flexores profundos da região cervical. ${ }^{(54)}$ Os dados obtidos indicam que a menor ativação dos músculos flexores profundos cervicais pode estar associada à presença de mudanças no tamanho muscular. ${ }^{(53)}$ Assim, o tamanho do músculo pode indicar uma medida indireta de força para a musculatura do pescoço. ${ }^{(55)}$

Pesquisas recentes com USG indicam que pacientes com dor cervical crônica apresentam medidas reduzidas do FLP, como a área de secção transversa (AST), quando comparados a sujeitos saudáveis. A diferença na AST entre os grupos é atribuída a uma possível atrofia no FLP em pacientes com dor crônica cervical.(47) Apesar das evidências encontradas sobre a importância dos músculos profundos no controle e suporte da lordose cervical e na manutenção do alinhamento da coluna cervical, ${ }^{(44-46)}$ a utilização da USG na avaliação destes músculos em pacientes com migrânea e CTT ainda é escassa. ${ }^{(44,47)}$

Outro método frequentemente utilizado na prática clínica de muitos profissionais para avaliar a atividade muscular é a eletromiografia (EMG). ${ }^{(44)}$ Nos dados obtidos pela EMG de superfície, a amplitude do sinal eletromiográfico é avaliada a partir dos valores da Root Mean Square (RMS), raiz quadrada da média dos quadrados da voltagem ao longo do ciclo. As alterações do espectro de potência do sinal eletromiográfico podem ser constatadas pelos valores de frequência média e mediana, em virtude de mudanças na taxa de disparo das unidades motoras. ${ }^{(56)}$ No caso dos músculos profundos, a capacidade contrátil do FLP e FLC pode ser avaliada por meio de EMG invasiva, via orofaríngea. ${ }^{(57)}$

Segundo alguns autores, em pacientes com dor cervical foi observada uma diminuição da atividade elétrica na EMG desta musculatura. ${ }^{(58)}$ Esta redução pode ser acompanhada da redução na eficiência neuromuscular dos músculos flexores superficiais, com atraso no início do relaxamento ${ }^{(59-60)}$ e maior fatigabilidade, ${ }^{(61)}$ aferidas através de EMG de superfície. ${ }^{(59-60)}$

Já em estudos com portadores de dor cervical associada à migrânea, ${ }^{(6)}$ os participantes foram submetidos à EMG de superfície do músculo ECM, na qual foi observado um aumento da atividade desta mus- 
culatura em compensação a menor ativação do músculo FLP. ${ }^{(61-63)}$ Os resultados sugerem que, em pacientes com cefaleia, há uma menor estabilidade da musculatura estabilizadora primária da região cervical e um aumento compensatório da atividade dos músculos superficiais quando comparados aos sujeitos do grupo controle. ${ }^{(61)}$

Pesquisas com EMG sugerem que, ao contrário do que se acreditava durante muito tempo, a CTT não é consequência apenas do estresse psicológico ou da contração muscular exacerbada da região cefálica e cervical. Da mesma forma, observou-se que a migrânea não envolve apenas mecanismos centrais. Tais suspeitas foram levantadas a partir da avaliação eletromiográfica de pacientes com e sem cefaleia. Estes estudos revelaram que alguns dos pacientes não tinham CTT ou tinham apenas migrânea episódica, mas apresentavam maior contração muscular. Por outro lado, os resultados também mostraram que, no mesmo grupo avaliado, alguns participantes tinham CTT grave, mas apresentavam um nível de contração muscular menor do que o grupo sem cefaleia. ${ }^{(16)}$ Portanto, é possível observar que alguns pacientes com cefaleia podem ter um aumento da atividade elétrica da musculatura cervical quando comparado a um grupo controle saudável, ${ }^{(64)}$ e outros casos nos quais se encontra uma atividade normal em ambos os grupos. ${ }^{(65)}$

Assim, existem disponíveis na literatura variados métodos de investigação das disfunções musculoesqueléticas cervicais em pacientes com dor, tal como a cefaleia. Neste contexto, cabe ressaltar que apesar de muitos estudos mostrarem que os métodos de investigação selecionados são eficazes na identificação das disfunções musculoesqueléticas, é preciso considerar alguns aspectos metodológicos antes de fazer inferências sobre os resultados encontrados. Portanto, os critérios de elegibilidade dos estudos, como as características dos participantes incluídos na amostra e os protocolos de avaliação utilizados devem ser previamente analisados. ${ }^{(15)}$

\section{CONSIDERAÇÕES FINAIS}

Estudos sugerem o envolvimento de mecanismos periféricos provenientes dos músculos cervicais na sensibilização do sistema nervoso central e no surgimento dos sintomas de dor na migrânea e na CTT. Os achados indicam que pacientes migranosos e com CTT podem apresentar alterações musculoesqueléticas cervicais, como o controle motor deficitário, modificando a estabilidade, a mobilidade e a biomecânica cervical. Todavia, muitos dos estudos que defendem a existência de inputs nociceptivos aferentes cervicais como facilitadores da cefaleia são baseados em estudos com animais, os quais apresentam sistemas de processamento da dor diferentes da espécie humana.

Apesar da grande variedade na literatura de métodos de investigação das disfunções musculoesqueléticas, os aspectos metodológicos das pesquisas analisadas comprometem a eficácia encontrada no resultado das mesmas. Ademais, há uma escassez de estudos utilizando métodos não invasivos e de baixo custo, como a USG e a EMG de superfície, na avaliação muscular em pacientes com migrânea e CTT.

Desta forma, sugere-se o delineamento de novos estudos com seres humanos, em uma população mais homogênea, analisando a associação entre a presença de migrânea e CTT e as disfunções musculoesqueléticas cervicais, com maior rigor metodológico e protocolos descritos de maneira mais detalhada e reprodutível, no intuito de direcionar o tratamento alternativo, não farmacológico, de pacientes com desordens musculares associadas a estas doenças.

\section{REFERÊNCIAS}

1. Gureje O, Von Korff M, Simon GE, Gater R. Persistent pain and well being: a World Health Organization study in primary care. JAMA. 1998;280(2):147-51.

2. Sokolovic E, Riederer F, Szucs T, Agosti R, Sándor PS. Self-reported headache among the employees of a Swiss university hospital: prevalence, disability, current treatment, and economic impact. J Headache Pain. 2013;14(1):1-8.

3. Bigal ME, Bigal JM, Betti M, Bordini CA, Speciali JG. Evaluation of the impact of migraine and episodic tension? type headache on the quality of life and performance of a university student population. Headache. $2001 ; 41$ (7):710-9.

4. Headache Classification Committee of the International Headache Society. The international classification of headache disorderes, 3rd edition (beta version). Cephalalgia. 2013; 33(9): 629-808.

5. Bekkelund SI, Salvesen R. Prevalence of head trauma in patients with difficult headache: the North Norway headache study. Headache. 2003;43(1):59-62.

6. Oksanen A, Erkintalo M, Metsähonkala L, Anttila P, Laimi K, Hiekkanen $\mathrm{H}$, et al. Neck muscles cross-sectional area in adolescents with and without headache-MRI study. Eur J Pain. 2008; 12(7):952-9.

7. Biondi DM. Physical treatments for headache: a structured review. Headache. 2005;45(6):738-46.

8. Hodges PW, Moseley GL. Pain and motor control of the lumbopelvic region: effect and possible mechanisms. J Electromyogr Kinesiol. 2003;13(4):361-70. 
9. Fernández-de-las-Peñas C, Alonso?Blanco C, Cuadrado M, Pareja J. Forward head posture and neck mobility in chronic tension-type headache: a blinded, controlled study. Cephalalgia. 2006;26(3):314-9.

10. Bendtsen L. Central sensitization in tension-type headachepossible pathophysiological mechanisms. Cephalalgia. 2000;20(5):486-508.

11. Holroyd KA. Behavioral and psychologic aspects of the pathophysiology and management of tension-type headache. Curr Pain Headache Rep. 2002;6(5):401-7.

12. Jensen R. Peripheral and central mechanisms in tension-type headache: an update. Cephalalgia. 2003;23 suppl 1:49-52.

13. Sohn J, Choi H, Lee S, Jun A. Differences in cervical musculoskeletal impairment between episodic and chronic tension-type headache. Cephalalgia. 2010;30(12):1514-23.

14. Burstein R. Deconstructing migraine headache into peripheral and central sensitization. Pain. 2001 ;89(2-3):107-10.

15. Robertson B, Morris M. The role of cervical dysfunction in migraine: a systematic review. Cephalalgia. 2008;28(5):474-83.

16. Krymchantowski AV. Cefaléias do tipo tensional. Migrâneas cefaléias, São Paulo. 2003;6(4):129-35.

17. Lipchik GL, Holroyd KA, Talbot F, Greer M. Pericranial muscle tenderness and exteroceptive suppression of temporalis muscle activity: a blind study of chronic tension-type headache. Headache. 1997;37(6):368-76.

18. Vandenheede M, Schoenen J. Central mechanisms in tensiontype headaches. Curr Pain Headache Rep. 2002;6(5):392 400.

19. Metsahonkala L, Anttila P, Laimi K, Aromaa M, Helenius H, Mikkelsson $M$, et al. Extracephalic tenderness and pressure pain threshold in children with headache. Eur J Pain. 2006; 10(7):581-5.

20. Fumal A, Schoenen J. Tension-type headache: current research and clinical management. Lancet Neurol. 2008;7(1):70-83.

21. Ashina S. Pathophysiology of tension-type headache. Curr Pain Headache Rep. 2005;9(6):415-22.

22. Wright EF. Referred craniofacial pain patterns in patients with temporomandibular disorder. J Am Dent Assoc. 2000;131 (9): 1307-15.

23. Wall PD, Woolf CJ. Muscle but not cutaneous C-afferent input produces prolonged increases in the excitability of the flexion reflex in the rat. J Physiol. 1984;356(1):443-58.

24. Fernández? de? las?Peñas C, Cuadrado ML, Pareja JA. Myofascial trigger points, neck mobility, and forward head posture in episodic tension-type headache. Headache. 2007;47(5):662-72.

25. Biondi DM. Cervicogenic headache: mechanisms, evaluation, and treatment strategies. J Am Osteopath Assoc. 2000;1009 suppl:7S-14S.

26. Bartsch T, Goadsby PJ. The trigeminocervical complex and migraine: current concepts and synthesis. Curr Pain Headache Rep. 2003;7(5):371-6.

27. Bartsch T. Migraine and the neck: new insights from basic data. Curr Pain Headache Rep. 2005;9(3):191-6.

28. Edvinsson L. Aspects on the pathophysiology of migraine and cluster headache. Pharmacol Toxicol. $2001 ; 89(2): 65-73$.
29. Russell MB. Genetic epidemiology of migraine and cluster headache. Cephalalgia. 2002;17(6):683-701.

30. Shevel E, Spierings EH. Cervical muscles in the pathogenesis of migraine headache. J Headache Pain. 2004;5(1):12-4.

31. Ji R-R, Kohno T, Moore KA, Woolf CJ. Central sensitization and LTP: do pain and memory share similar mechanisms? Trends Neurosci. 2003;26(12):696-705.

32. Magerl W, Fuchs PN, Meyer RA, Treede R-D. Roles of capsaicininsensitive nociceptors in cutaneous pain and secondary hyperalgesia. Brain. 2001;124(9):1754-64.

33. Wood J. Recent advances in understanding molecular mechanisms of primary afferent activation. Gut. 2004;53 suppl 2:ii9-ii 12.

34. Woolf CJ, Salter MW. Neuronal plasticity: increasing the gain in pain. Science. 2000;288(5472): $1765-9$

35. Sanchez RM, Reuter U, Moskowitz M. Central and peripheral mechanisms of migraine. Funct Neurol. 2000; 15(3):157-62.

36. Gebhart G. Descending modulation of pain. Neurosci Biobehav Rev. $2004 ; 27(8):$ 729-37.

37. Pogatzki EM, Gebhart G, Brennan TJ. Characterization of A? and $\mathrm{C}$-fibers innervating the plantar rat hindpaw one day after an incision. J. Neurophysiol. 2002;87(2):721-31.

38. Bartsch T, Goadsby P. Increased responses in trigeminocervical nociceptive neurons to cervical input after stimulation of the dura mater. Brain. 2003;126(8):1801-13.

39. Jensen R, Rasmussen BK, Pedersen B, Olesen J. Muscle tenderness and pressure pain thresholds in headache. A population study. Pain. 1993;52(2): 193-9.

40. Sjaastad $\bigcirc$, Fredriksen T, Pfaffenrath V. Cervicogenic headache: diagnostic criteria. Headache. 1998;38(6):442-5.

41. Fernández-de-las-Peñas C, Cuadrado M, Pareja J. Myofascial trigger points, neck mobility and forward head posture in unilateral migraine. Cephalalgia. 2006;26(9):1061-70.

42. Fernández? de? las? Peñas C, Falla D, Arendt-Nielsen L, Farina D. Cervical muscle co?activation in isometric contractions is enhanced in chronic tension?type headache patients. Cephalalgia. 2008;28(7):744-51.

43. Bovim G, Sand T. Cervicogenic headache, migraine without aura and tension-type headache. Diagnostic blockade of greater occipital and supra-orbital nerves. Pain. 1992;51 (1):43-8.

44. Cagnie B, Derese E, Vandamme L, Verstraete K, Cambier D, Danneels L. Validity and reliability of ultrasonography for the longus colli in asymptomatic subjects. Man Ther. 2009; 14(4):421-6.

45. Boyd-Clark L, Briggs C, Galea M. Comparative histochemical composition of muscle fibres in a pre- and a postvertebral muscle of the cervical spine. J Anat. 2001;199(6):709-16.

46. Boyd-Clark L, Briggs C, Galea M. Muscle spindle distribution, morphology, and density in longus colli and multifidus muscles of the cervical spine. Spine. 2002;27(7):694-701.

47. Javanshir K, Mohseni-Bandpei MA, Rezasoltani A, Amiri M, Rahgozar M. Ultrasonography of longus colli muscle: A reliability study on healthy subjects and patients with chronic neck pain. J Bodyw Mov Ther. $2011 ; 15(1): 50-6$.

48. Falla D. Unravelling the complexity of muscle impairment in chronic neck pain. Man Ther. 2004;9(3):125-33. 
49. O'Leary S, Jull G, Kim M, Vicenzino B. Cranio-cervical flexor muscle impairment at maximal, moderate, and low loads is a feature of neck pain. Man Ther. 2007;12(1):34-9.

50. Griegel-Morris P, Larson K, Mueller-Klaus K, Oatis CA. Incidence of common postural abnormalities in the cervical, shoulder, and thoracic regions and their association with pain in two age groups of healthy subjects. Phys Ther. 1992;72(6):425-31.

51. Stokes M, Hides J, Elliott J, Kiesel K, Hodges P. Rehabilitative ultrasound imaging of the posterior paraspinal muscles. J Orthop Sports Phys Ther.. 2007;37(10):581-95.

52. Elliott J, Jull G, Noteboom JT, Galloway G. MRI study of the cross-sectional area for the cervical extensor musculature in patients with persistent whiplash associated disorders (WAD). Man Ther. 2008;13(3):258-65.

53. Javanshir K, Amiri M, Mohseni-Bandpei MA, Rezasoltani A, Fernández-de-las-Peñas $C$. Ultrasonography of the cervical muscles: a critical review of the literature. J Manipulative Physiol Ther. 2010;33(8):630-7.

54. Jesus-Moraleida FR, Ferreira PH, Pereira LSM, Vasconcelos CM, Ferreira ML. Ultrasonographic analysis of the neck flexor muscles in patients with chronic neck pain and changes after cervical spine mobilization. J Manipulative Physiol Ther. 2011 ;34(8):514-24.

55. Rezasoltani A, Ylinen J, Vihko V. Isometric cervical extension force and dimensions of semispinalis capitis muscle. J Rehabil Res Dev. 2002;39(3):423-8.

56. Merletti R, Rainoldi A, Farina D. Surface electromyography for noninvasive characterization of muscle. Exerc Sport Sci Rev. $2001 ; 29(1): 20-5$.

57. Falla D, Jull G, Dall'Alba P, Rainoldi A, Merletti R. An electromyographic analysis of the deep cervical flexor muscles in performance of craniocervical flexion. Phys Ther. 2003; 83(10):899-906.

58. Falla D, Jull G, Hodges P. Feedforward activity of the cervical flexor muscles during voluntary arm movements is delayed in chronic neck pain. Exp Brain Res. 2004; 157(1):43-8.
59. Jull G, Kristjansson E, Dall'Alba P. Impairment in the cervical flexors: a comparison of whiplash and insidious onset neck pain patients. Man Ther. 2004;9(2):89-94.

60. Javanshir K, Rezasoltani A, Mohseni-Bandpei MA, Amiri M, Ortega-Santiago R, Fernández-de-Las-Peñas C. Ultrasound assessment of bilateral longus colli muscles in subjects with chronic bilateral neck pain. Am J Phys Med Rehabil. 2011;90 (4):293-301.

61. Falla D, Rainoldi A, Merletti R, Jull G. Myoelectric manifestations of sternocleidomastoid and anterior scalene muscle fatigue in chronic neck pain patients. Clin Neurophysiol. 2003;1 14(3): 488-95.

62. Sterling M, Jull G, Wright A. Cervical mobilisation: concurrent effects on pain, sympathetic nervous system activity and motor activity. Man Ther. $2001 ; 6(2): 72-81$.

63. Falla D, Jull G, Edwards S, Koh K, Rainoldi A. Neuromuscular efficiency of the sternocleidomastoid and anterior scalene muscles in patients with chronic neck pain. Disabil Rehabil. 2004;26(12):712-7.

64. Jensen R, Fuglsang-Frederiksen A, Olesen J. Quantitative surface EMG of pericranial muscles in headache. A population study. Electroencephalogr Clin Neurophysiol. 1994;93(5):335-44.

65. Rollnik JD, Karst M, Fink M, Dengler R. Botulinum toxin type A and EMG: A key to the understanding of chronic tension-type headaches? Headache. $2001 ; 41$ (10):985-9.

Correspondência

Daniella Araújo de Oliveira.

Departamento de Fisioterapia Universidade Federal de Pernambuco Av. Jornalista Anibal Fernandes, s/n, Cidade Universitária 50740-560, Recife - PE

Tel.: (81) 21268490 sabino_daniella@ig.com.br 